\title{
A gene-based information gain method for detecting gene-gene interactions in case-control studies
}

\author{
Jin $\mathrm{Li}^{1,2,3,5}$, Dongli Huang ${ }^{1,5}$, Maozu Guo ${ }^{\star, 1}$, Xiaoyan Liu ${ }^{1}$, Chunyu Wang ${ }^{1}$, Zhixia Teng ${ }^{1}$, Ruijie Zhang ${ }^{3}$, \\ Yongshuai Jiang ${ }^{3}$, Hongchao $\mathrm{Lv}^{3}$ and Limei Wang ${ }^{\star, 3,4}$ \\ Currently, most methods for detecting gene-gene interactions (GGIs) in genome-wide association studies are divided into \\ SNP-based methods and gene-based methods. Generally, the gene-based methods can be more powerful than SNP-based \\ methods. Some gene-based entropy methods can only capture the linear relationship between genes. We therefore proposed a \\ nonparametric gene-based information gain method (GBIGM) that can capture both linear relationship and nonlinear correlation \\ between genes. Through simulation with different odds ratio, sample size and prevalence rate, GBIGM was shown to be valid \\ and more powerful than classic KCCU method and SNP-based entropy method. In the analysis of data from 17 genes on \\ rheumatoid arthritis, GBIGM was more effective than the other two methods as it obtains fewer significant results, which was \\ important for biological verification. Therefore, GBIGM is a suitable and powerful tool for detecting GGls in case-control studies. \\ European Journal of Human Genetics (2015) 23, 1566-1572; doi:10.1038/ejhg.2015.16; published online 11 March 2015
}

\section{INTRODUCTION}

Genome-wide association studies (GWAS) have rapidly become a popular and powerful tool for human disease-associated gene discovery. ${ }^{1}$ Many single-SNP-based GWAS methods have emerged for several years. ${ }^{1,2}$ However, due to the huge number of human genome SNPs (hundreds of thousands or millions in a test), the statistical power and efficiency of these methods are limited. ${ }^{3}$ In addition, human complex diseases are generally caused by the combined effect of multiple genes, and a single SNP is difficult to explain the pathogenesis of diseases. ${ }^{4,5}$ The detection of interactions between genes is important to gain a better understanding of the genetic mechanisms of human complex diseases.

Large numbers of SNP-SNP interactions (SSIs) detecting methods appeared in recent years. In a case-control study, one form of SSI (or named co-associations) was epistasis, which was introduced $\sim 100$ years ago. These SSIs are associated with gene-gene interactions (GGIs). The GGIs (or gene-gene epistasis) are often characterized to be functional, compositional and statistical. ${ }^{6}$ The statistical definition of epistasis was first given by Fisher $^{7}$ and developed further by Cockerham $^{8}$ and Kempthorne, ${ }^{9}$ whereby the epistasis effect is considered as a deviation from additive genetic effects. ${ }^{10}$ Currently, popular SSIs detecting methods are based primarily on statistics, ${ }^{11-13}$ data mining, ${ }^{14-16}$ machine learning ${ }^{17,18}$ and so on. ${ }^{19,20}$ Statistical methods contain logistic regression model, ${ }^{11}$ the information entropy model; ${ }^{12,13}$ data mining methods contain dimensionality reduction method, ${ }^{14}$ Bayesian method ${ }^{15,16}$ and so on; machine learning methods are based on a tree and random forests ${ }^{17,18}$ and so on.

We take only one SNP in a gene as a basic research unit, and only take into account the interactions between SNPs in these SSI methods.
However, a gene that contains many SNPs should be the basic research unit, so the SSIs have limitations and cannot fully interpret the GGIs. ${ }^{21}$ We can use multiple SNPs in each gene (gene-based GGI methods), and these methods come with a potential increase of power.

There are some gene-based GGI detecting methods, such as canonical correlation-based $U$-statistic model, ${ }^{21}$ sparse canonical correlation analysis model, ${ }^{22}$ kernel canonical correlation-based $U$-statistic model (KCCU), ${ }^{23,24}$ kernel regression model (KR), ${ }^{25}$ partial least squares path model (PLSPM and MPLSPM) ${ }^{26,27}$ and so on. However, most of these methods can only reflect the linear relationship between two genes, and cannot reflect the nonlinear relationship. KCCU method can reflect nonlinear relationship between two genes, and is a useful and classic method.

Information entropy ${ }^{28,29}$ is used to measure the uncertainty. The greater the uncertainty variables, the greater the entropy. The SNPbased entropy methods (SBEM) had been used to detect SSIs. ${ }^{12,13}$ We proposed a gene-based information gain method (GBIGM), which is based on the entropy and information gain theory and views all SNPs in a gene for detecting GGIs in case-control studies. For a gene, we defined an information gain rate by comparing the entropy of the data with and without a gene's information. We consider IGR as a measure of genetic contribution for disease for this gene. While considering two genes, the IGR can be determined by comparing the joint entropy and individual entropies and we use it as a measure for epistasis. After comparing GBIGM with KCCU and SBEM both in the simulated and real data, we found that GBIGM can detect several epistasis types, and it is a valid and powerful gene-based method for detecting GGIs.

${ }^{1}$ Nature Computation Lab, School of Computer Science and Technology, Harbin Institute of Technology, Harbin, China; ${ }^{2}$ School of Life Science and Technology, Harbin Institute of Technology, Harbin, China; ${ }^{3}$ Department of Statistics Genetics, College of Bioinformatics Science and Technology, Harbin Medical University, Harbin, China; ${ }^{4}$ Center of Computer Science, School of Basic Medical Sciences, Harbin Medical University, Harbin, China

*Correspondence: Professor M Guo, School of Computer Science and Technology, Harbin Institute of Technology, NO. 92, West Dazhi Street, Nan Gang District, Harbin, 150001 China. Tel/Fax: +86 45186402407 ; E-mail: maozuguo@hit.edu.cn

or Dr L Wang, College of Bioinformatics Science and Technology, Harbin Medical University, NO. 157, Road Baojian, Nan Gang District, Harbin, 150086 China. Tel/Fax: +86 451 86667543; E-mail: wlm@hrbmu.edu.cn

${ }^{5}$ Co-first authors.

Received 28 September 2014; revised 30 November 2014; accepted 14 January 2015; published online 11 March 2015 


\section{MATERIALS AND METHODS}

\section{Simulation data}

Get the template data. The template data in simulation experiments are taken from HapMap. ${ }^{30,31}$ In this study, we randomly select two gene regions, ${ }^{23}$ PARP1 and KRAS. PARP1 is located on chromosome 1, including seven SNPs (rs7537552, rs7537636, rs10495278, rs9287011, rs12090413, rs12092786 and rs12093044). KRAS is located on chromosome 12, including five SNPs (rs3924649, rs12307733, rs7980769, rs11836162 and rs11047882)

Disease models. A disease model is a model that expresses the relationship between the gene and the disease. ${ }^{32}$ Disease models are usually divided into two types, namely, single-site and multi-locus disease models. In single-site disease models, the disease is linked to only one locus, and in multi-locus model the disease is related to multiple loci.

This paper concentrated on the interaction between two genes, so we generate case-control data with two-locus disease model. There are eight twolocus disease models when generating simulation data using gs 2.0 software, ${ }^{33}$ as showed in Supplementary Figure 1.

In Supplementary Figure 1, $\alpha$ is the baseline value, which indicates the odds of disease when the two SNP's joint genotype is aabb. $\theta$ is the growth leading to illness when other genotype with respect to aabb.

Generate case-control data sets. In this study, we utilize all these eight twolocus disease models in gs 2.0 program to generate simulated case-control data. In the simulation, one SNP in each gene is randomly selected to set the parameter in generating case-control data, and other SNPs are generated using the information of linkage disequilibrium. The parameter settings are given in Table 1.

In case of the null hypothesis $H_{0}$ (to generate negative data sets), there is no interaction between genes, then the odds ratio (OR) was set to be 1.0. We set same sample size for cases and controls $(N$ cases and $N$ controls, $N$ is set to be $1000,2000,3000,4000$ and 5000 ) and prevalence rate to be 0.1 .

In the case of the alternative hypothesis $H_{1}$ (to generate positive data sets), to test the effects of OR, sample size and prevalence rate respectively in GGIs detecting, we set three scenarios to perform experiments. In the first scenario, we set $\mathrm{OR}$ to be $1.2,1.4,1.6,1.8,2.0$ and 2.2, sample sizes to be 2000 and prevalence rate to be 0.1 . In the second scenario, we set OR to be 1.6 , sample size $\mathrm{N}$ to be $1000,2000,3000,4000$ and 5000 and prevalence rate to be 0.1 . In the third scenario, we set OR to be 1.6, sample size $\mathrm{N}$ to be 2000 and prevalence rates to be $0.01,0.05,0.10,0.15$ and 0.20 . For each parameter setting, we performed the experiment 100 times.

\section{Rheumatoid arthritis data set}

We apply our method to a rheumatoid arthritis (RA) data set (GSE39428). ${ }^{34}$ The data set contains 266 cases (RA) and 163 health controls. Genotyping is performed using a custom-designed Illumina 384-SNP VeraCode microarray (Illumina, San Diego, CA, USA) to determine possible associations of genes to RA. After pretreatment, we obtain 381 SNPs encoding 17 genes.

\section{GBIGM}

In a case-control study, we assume that there are $N_{\text {case }}$ cases and $N_{\text {control }}$ controls. For arbitrary two genes $G_{1}$ and $G_{2}$, there are k SNPs in gene $\mathrm{G}_{1}$ and $\mathrm{t}$ SNPs in gene $\mathrm{G}_{2}$. The framework of the GBIGM is described as follows.

Table 1 The parameter settings in simulation using gs2.0

\begin{tabular}{llcc}
\hline & Odds ratio & Sample size & Prevalence rate \\
\hline Negative data sets & 1.0 & $1000 \sim 5000$ & 0.1 \\
Positive data sets I & $1.2 \sim 2.2$ & 2000 & 0.1 \\
Positive data sets II & 1.6 & $1000 \sim 5000$ & 0.1 \\
Positive data sets III & 1.6 & 2000 & $0.01 \sim 0.2$ \\
\hline
\end{tabular}

\section{ALGORITHM: GBIGM}

Input: SNPs on two genes $\left(\mathrm{G}_{1}\right.$ and $\left.\mathrm{G}_{2}\right)$ in a case-control study, times of permutation $m$. Output: $P$-value

Step 1: Compute the entropy $H_{0}$ of initial data set.

Step 2: Compute the conditional entropy and information gain rate for gene $G_{1}, G_{2}$. Step 3: Compute conditional entropy $H_{1,2}$ and information gain rate $\Delta R_{1,2}$ for gene $\mathrm{G}_{1}$ and $\mathrm{G}_{2}$

Step 4: Perform relabeling and generate new data set.

Step 5: Repeat steps (2) to (5) $m$ times.

Step 6: Estimate $P$-value.

The detailed description is as follows.

Step 1: Compute the entropy $H_{0}$ of initial data set

In the initial data set $D$, the samples are divided either cases or controls, and $p$ (case) is the proportion of cases in $D$, which is calculated

$$
p(\text { case })=\frac{N_{\text {case }}}{N_{\text {case }}+N_{\text {control }}}
$$

$H(\bullet)$ is defined as a classic entropy function, and the entropy $H_{0}$ of $D$ can be defined as

$$
\begin{aligned}
H_{0} & =H(D) \\
& =-p(\text { case }) \log (p(\text { case }))-(1-p(\text { case })) \log (1-p(\text { case }))
\end{aligned}
$$

Step 2:

(1) Compute the conditional entropy $H_{1}$ and information gain rate $\Delta R_{1}$ for gene $\mathrm{G}_{1}$

The $k$ SNPs in gene $\mathrm{G}_{1}$ are quantified as $X$,

$$
X=\left(x_{1}, x_{2} \ldots, x_{k}\right) x_{i} \in\{0,1,2\} i=1,2, \ldots k
$$

The conditional entropy $H_{1}$, information gain $I G(D \backslash X)$ and information gain rate $\Delta R_{1}$ for gene $\mathrm{G}_{1}$ are defined as follows:

$$
\begin{gathered}
H_{1}=H(D \mid X)=H(D, X)-H(X) \\
=H\left(D, x_{1}, x_{2}, \cdots x_{k}\right)-H\left(x_{1}, x_{2}, \cdots x_{k}\right) \\
I G(D \mid X)=H_{0}-H_{1} \\
\Delta R_{1}=\frac{H_{0}-H_{1}}{H_{0}}
\end{gathered}
$$

Here the conditional entropy $H_{1}$ of $D$ conditioned on $X$ can be calculated as the difference between joint entropy of $D$ and $X$ and entropy of $X$. For any $D$ and $X$, $H(D \backslash X) \leq H(D)$ constant sets up. So the information gain $I G(D \backslash X)$ describes the difference value of entropies conditioned on $X$ or not, which can reflect the importance of $X$. The information gain rate $\Delta R_{1}$ is a normalized information gain.

(2) Compute the conditional entropy $H_{2}$ and information gain rate $\Delta R_{2}$ for gene $\mathrm{G}_{2}$

The $t$ SNPs in gene $\mathrm{G}_{2}$ are quantified as $Y$,

$$
Y=\left(y_{1}, y_{2}, \cdots, y_{t}\right) y_{j} \in\{0,1,2\} j=1,2, \cdots, t
$$

Similarly, the conditional entropy $H_{2}$, information gain $I G(D \backslash Y)$ and information gain rate $\Delta R_{2}$ for gene $G_{2}$ are defined as follows:

$$
\begin{gathered}
H_{2}=H(D \mid Y)=H(D, Y)-H(Y) \\
=H\left(D, y_{1}, y_{2}, \cdots, y_{t}\right)-H\left(y_{1}, y_{2}, \cdots, y_{t}\right) \\
I G(D \mid Y)=H_{0}-H_{2} \\
\Delta R_{2}=\frac{H_{0}-H_{2}}{H_{0}}
\end{gathered}
$$

Step 3: Compute conditional entropy $H_{1,2}$ and information gain rate $\Delta R_{1,2}$ for gene $G_{1}$ and $G_{2}$

$$
\begin{gathered}
H_{1,2}=H(D \mid X, Y)=H(D, X, Y)-H(X, Y) \\
\Delta R_{1,2}=\frac{\min \left\{H_{1}, H_{2}\right\}-H_{1,2}}{\min \left\{H_{1}, H_{2}\right\}}
\end{gathered}
$$


$\Delta R_{1,2}$ is the normalized difference between the entropy conditioned on $X$ and $Y$ and the entropy conditioned on $X$ or $Y$ (the minimum between entropy conditioned on $X$ and entropy conditioned on $Y$ ). It represents the normalized information gain while considering both $X$ and $Y$ and considering only $X$ or $Y$. Therefore, the larger the $\Delta R_{1,2}$, the larger probability of epistasis between $X$ and $Y$.

In the initial data, we calculate statistics $\Delta R_{1,2}$ and denote it as $\Delta R_{1,2}^{0}$.

Step 4: Perform relabeling and generate new data set

As the model does not assume any distribution of the data, it is difficult to use conventional parametric test for significant inference. In this study, we use a displacement detection test method (Permutation) ${ }^{35,36}$ for detecting significant GGIs. In the permutation test, we relabel the samples to generate a new random case and control groups, then recalculate the statistic, construct the empirical distribution and finally estimate $P$-values.

Step 5: Repeat steps (2) to (4) $m$ times

For a given number $(m)$ of permutation times, repeat steps (2) to (4) $m$ times. We will obtain $m$ statistics $\Delta R_{1,2}$, and we denote them as $\Delta R_{1,2}^{1}, \Delta R_{1,2}^{2}, \cdots, \Delta R_{1,2}^{m}$.

Step 6: Estimate $P$-value

Define the null hypothesis, alternative hypothesis and significance level.

$$
H_{0}: \Delta R_{1,2}=0 H_{1}: \Delta R_{1,2}>0 \alpha=0.05
$$

a
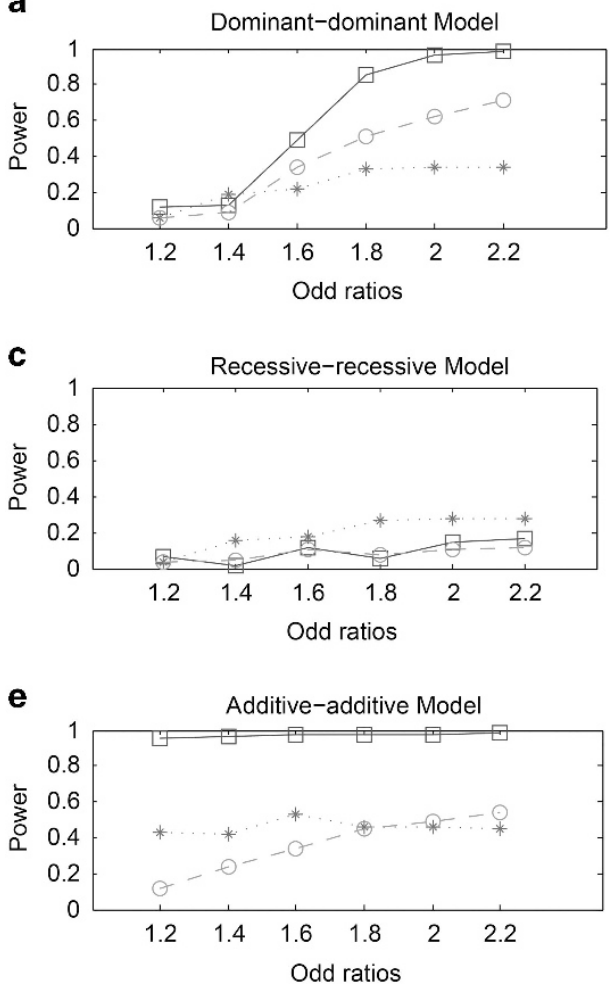

g

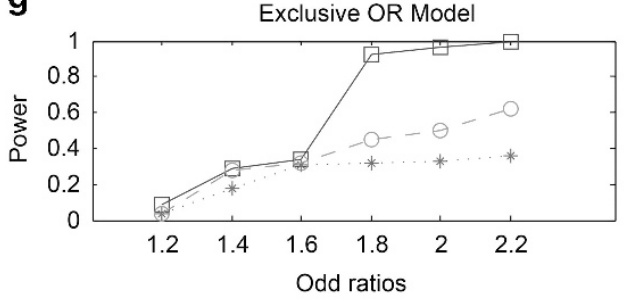

While we perform the permutation, the random samples are following the null hypothesis $H_{0}$. Therefore, according to $m$ statistics from random permutation samples, we can get the experience sampling distribution (empirical distribution) for the statistics $\Delta R_{1,2}$ following the null hypothesis $H_{0}$.

We count the number of statistics $\Delta R_{1,2}^{i}$ that will be equal to or greater than $\Delta R_{1,2}^{0}$.

$$
\begin{gathered}
\text { num }=\sum_{i=1}^{m} I\left(\Delta R_{1,2}^{i} \geq \Delta R^{0}{ }_{1,2}\right), \\
I\left(\Delta R_{1,2}^{i} \geq \Delta R_{1,2}^{0}\right)=\left\{\begin{array}{ll}
1 & \Delta R_{1,2}^{i} \geq \Delta R^{0}{ }_{1,2} \\
0 & \Delta R^{i}{ }_{1,2}<\Delta R_{1,2}^{0}
\end{array},\right.
\end{gathered}
$$

here $/(\cdot)$ is an indicator function.

Then the $P$-value can be estimated as

$$
P=\frac{\text { num }}{m}
$$

\section{SBEM}

The SBEM has been used to detect SSIs. ${ }^{12,13}$ We use one SNP as a representative in a gene each time, then calculate the entropy-based statistic and estimate the significance similar to GBIGM; at last we select the most

b

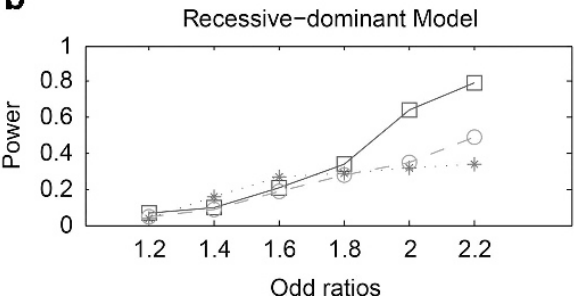

d

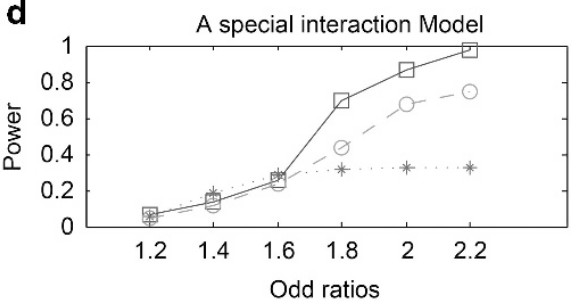

f

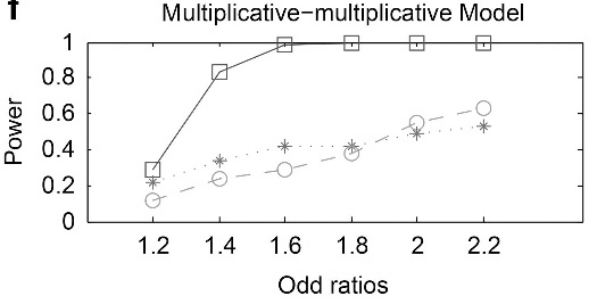

h

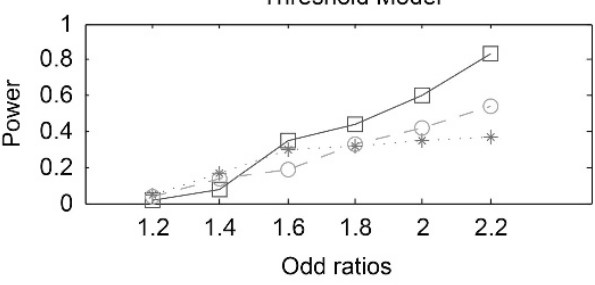

$-\square$ GBIGM

SBEM

KCCU

Figure 1 The power comparison among three different GGls detecting methods under different disease models and different OR values. The horizontal axis is the odd ratios ranging in 1.2 to 2.2 and the vertical axis is the power obtained from the three methods. There are 8 different disease models from $\mathbf{a}$ to $\mathbf{h}$. 
significant SSI result as the significance of GGI. So SBEM is a univariate SNP method and GBIGM is a multiple SNPs method. The detailed description of SBEM can be found in the paper of Dong et al. ${ }^{12}$

\section{$\mathrm{KCCU}$}

The kernel canonical correlation-based $U$-statistic model $(\mathrm{KCCU})^{23,24}$ can reflect nonlinear relationship between two genes and is a useful and classic method. In KCCU, the maximum kernel canonical coefficient of the two genes is taken as a measure of GGI in cases and controls. Let the genotyped data of case-control study be $\left(x_{1}^{D}, x_{2}^{D}, \cdots, x_{k}^{D}\right)$ and $\left(y_{1}^{D}, y_{2}^{D}, \cdots, y_{t}^{D}\right)$ for gene $\mathrm{G}_{1}$ and gene $\mathrm{G}_{2}$ for cases, and $\left(x_{1}^{C}, x_{2}^{C}, \cdots, x_{k}^{C}\right)$ and $\left(y_{1}^{C}, y_{2}^{C}, \cdots, y_{t}^{C}\right)$ for controls. The maximum kernel canonical coefficient $k r_{D}$ between $\left(x_{1}^{D}, x_{2}^{D}, \cdots, x_{k}^{D}\right)$ and $\left(y_{1}^{D}, y_{2}^{D}, \cdots, y_{t}^{D}\right)$ obtained through kernal canonical correlation analysis (KCCA) could be considered as a measurement of gene-based GGI in cases and $k r_{\mathrm{c}}$ between $\left(x_{1}^{C}, x_{2}^{C}, \cdots, x_{k}^{C}\right)$ and $\left(y_{1}^{C}, y_{2}^{C}, \cdots, y_{t}^{C}\right)$ be a measurement of GGI in controls. After a transformation to $k r_{D}$ and $k r_{\mathrm{c}}$ analogous to Fisher's simple correlation coefficient transformation, we can obtain a KCCU statistic. The detailed description of KCCU can be found in the paper of Yuan et al. ${ }^{23}$

\section{Evaluation indexes}

To test the effectiveness of GBIGM, we select power and false positive rate (Type I error probability) $p_{a}$ as evaluation indexes, and perform a comparative analysis with $\mathrm{SBEM}^{12}$ and KCCU. ${ }^{23}$
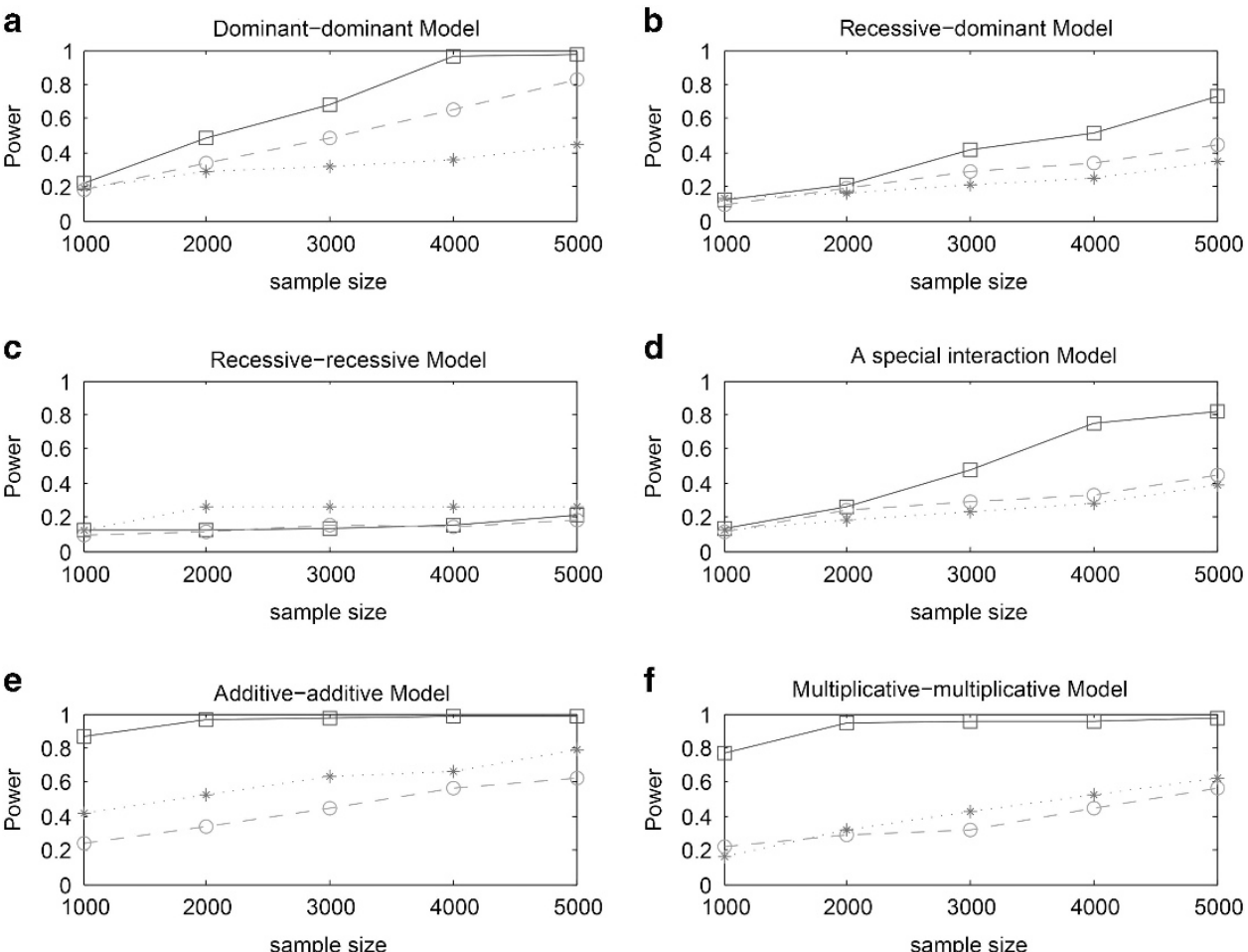

For GBIGM and KCCU, if the number of the $P$-values less than $\alpha$ is $m_{2}$, the power can be calculated as follows:

$$
\text { power }_{2}=\frac{m_{2}}{m}
$$

f

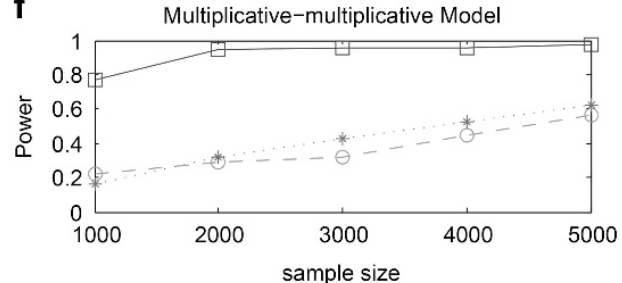

$\mathbf{h}$

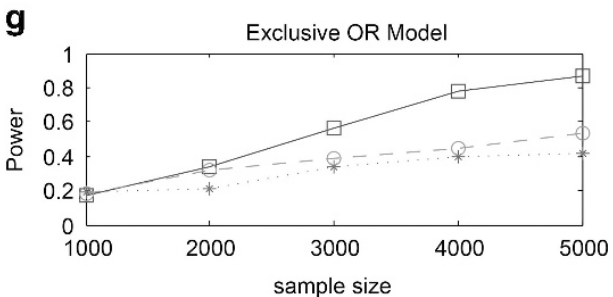

Power. The power of a statistical test is the probability that it correctly rejects the null hypothesis when the null hypothesis is incorrect (the alternative ypothesis is true). In this study, we performed the simulation $\mathrm{m}(100)$

For SBEM, when testing the GGIs, we need to compute all the SSIs between the two genes. Therefore, we need to make a multiple testing adjustment. Here we made use of the Bonferroni adjustment method. ${ }^{37}$ Assume the significance $P$-values. If the number of $P$-values less than $\alpha$ is $m_{1}$, the power of SBEM can be calculated as follows:

$$
\text { power }_{1}=\frac{m_{1}}{k \times t \times m}
$$


False positive rate $P_{\alpha}$. False positive rate $P_{\alpha}$ is the probability that it falsely rejects the null hypothesis when the null hypothesis is true, and it is also known as the probability of committing a Type I error. At a specific significance level $(\alpha=0.05)$, we perform the simulation 100 times with each different sample sizes when the null hypothesis is true. The observed $P_{\alpha}$ changes when the sample size changes from small to large. When $P_{\alpha}$ is stable near the significance level $\alpha$, we consider that the sample size is large enough to obtain a robust test result.

\section{RESULTS}

\section{Power}

The effect of $O R$. When OR value changes from 1.2 to 2.2, the power of GBIGM, SBEM and KCCU are indicated as Figure 1.

With the increasing OR value, the power of GBIGM, SBEM and KCCU are monotonously increasing under seven different genetic models except the recessive-recessive model. The power of GBIGM is significantly higher than the power of SBEM and KCCU under seven different genetic models except the recessive-recessive model. When the OR value reaches 1.8, under the five disease models of the dominant-dominant model, a special interaction model, multiplicative-multiplicative model, exclusive OR model and additive-additive model, the power of GBIGM has reached $80 \%$, whereas the power of
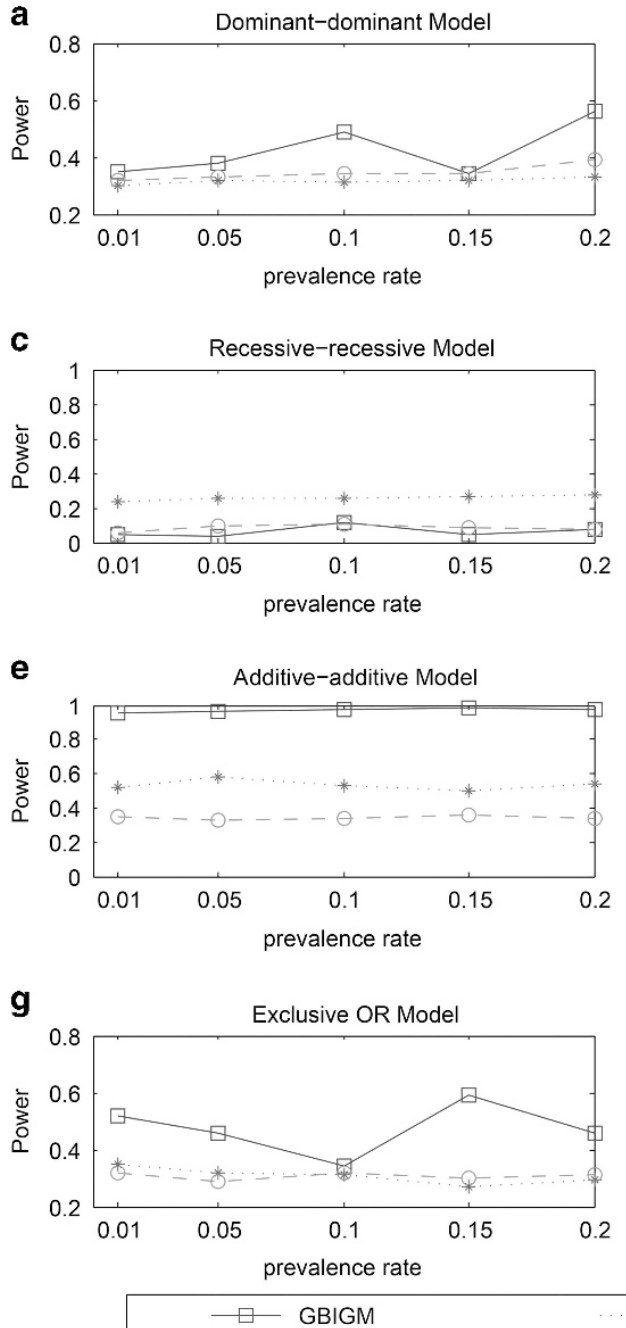

SBEM and KCCU are only about $40 \%$. Under the recessive-recessive model, when the OR value gradually changes from 1.2 to 2.2 , the power of the three GGIs detecting models are consistently below $20 \%$, which indicates that these three GGIs detecting models are unsuitable when the disease data conform with the recessive-recessive model.

The effect of sample size. When sample size changes from 1000 to 5000, the power of GBIGM, SBEM and KCCU are shown in Figure 2.

With an increase in the sample size, the power of GBIGM, SBEM and KCCU are monotonously increasing under seven different genetic models except the recessive-recessive model. The power of GBIGM is significantly higher than the power of SBEM and KCCU under seven different genetic models except the recessive-recessive model. When the sample size reaches 4000, under the six disease models of the dominant-dominant model, a special interaction model, multiplicative-multiplicative model, exclusive OR model, addictive-addictive model and threshold model, the power of GBIGM have reached $80 \%$, whereas the power of SBEM and KCCU are only about $40 \%$. Under the recessive-recessive model, when the sample size gradually changes from 1000 to 5000, the power of the three GGIs detection model are consistently below 20\%, which indicates that these three GGIs
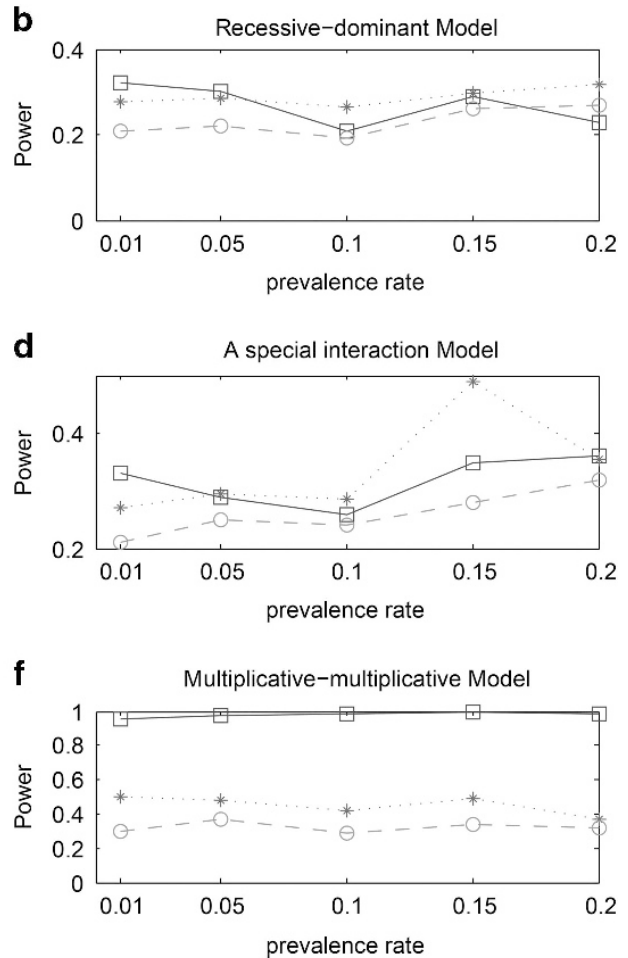

h

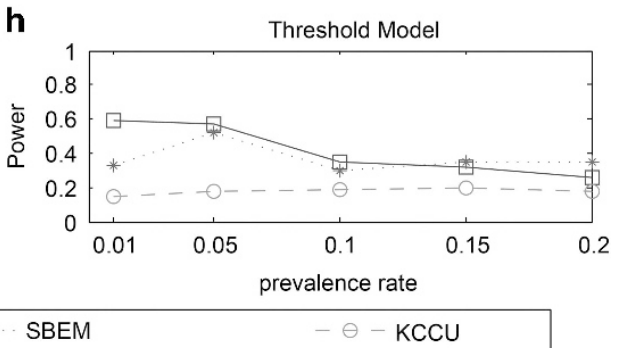

Figure 3 The power comparison among three different GGls detecting methods under different disease models and different prevalence rates. The horizontal axis is the prevalence rate ranging in 0.01 to 0.2 and the vertical axis is the power obtained from the three methods. There are 8 different disease models from a to $\mathbf{h}$. 
detecting models are unsuitable when the disease data conforms with the recessive-recessive model.

The effect of prevalence rate. When the prevalence rate changes from 0.01 to 0.20 , the power of GBIGM, SBEM and KCCU are shown in Figure 3.

From the results, we can see that there are no significant correlations between the power and prevalence rate, which indicate these GGIs detecting methods are not influenced from the prevalence rate. In the recessive-recessive model, the addictive-addictive model, the multiplicative-multiplicative model and the exclusive model, the power of GBIGM are significantly higher than the power of SBEM and KCCU. There are no significant differences between the power of these three methods in other models.

From the comparison of the power of these methods under different disease models and parameter settings, we conclude that all of these three methods are unsuitable for detecting GGIs when the interactions are the recessive-recessive model type. In other disease types, GBIGM is significantly powerful than SBEM and KCCU.

\section{False positive rates}

Set significance level $\alpha=0.05$, when the sample size gradually changes from 1000 to 5000 , the false positive rate $P_{\alpha}$ of GBIGM are shown in Table 2.

When the sample size changes from 1000 to 5000 gradually, false positive rate $P_{\alpha}$ are stable nearby the significance level $\alpha=0.05$ under seven different genetic models except the additive-additive model. It indicates that GBIGM is unsuitable for detecting GGIs when the interaction is the additive-additive model type. The results confirmed the stability of the model in most disease models.

GBIGM does not depend on any statistical distribution or model, so it could detect both the linear relationship and the nonlinear relationship between two genes. Integrate the power analysis and false positive rate analysis, we believe that GBIGM we proposed is a valid and robust method for detecting GGIs under six disease models except additive-additive model and recessive-recessive model.

\section{Applications in RA data}

In these data, the genes and SNP numbers in these genes are shown in Table 3. The numbers of SNPs in genes vary from 2 to 128 . We applied GBIGM, SBEM and KCCU in this data set for detecting GGIs related to RA.

In GBIGM and KCCU, we set the significance level $\alpha=0.05$, and obtained $5(3.68 \%)$ and $76(55.88 \%)$ significant GGIs, respectively. In SBEM, for each gene-gene pair, we took the minimum SNP-SNP $P$-value as the gene-gene $P$-value, and we obtained $123(90.44 \%)$ significant GGIs at the significance level $\alpha=0.05$ or $74(54.41 \%)$ significant GGIs at the significance level $\alpha=0.001$. The detailed results are presented in Supplementary Table 1. As the total number of genegene pair is 136, we obtained too many significant results when using SBEM and KCCU. The GBIGM method we proposed can significantly decrease the number of significant results, which is very important for the biological verification.

Of the five significant GGIs detected by GBIGM, they were also detected in KCCU, and the ranks of $P$-values were 33, 38, 24, 10 and 13. Especially, in these 17 genes, there was only one gene (PADI4) confirmed to be related to RA by OMIM. ${ }^{38,39}$ We detected a potential interaction between PADI4 and BUB3 in GBIGM ( $P$-value $=0.038$, rank 4$)$ and $\mathrm{KCCU}(P$-value $=9.62 \mathrm{E}-13$, rank 10), but not in SBEM at the significance level $\alpha=0.001$ ( $P$-value $=0.034$, rank 118). The ranks of these five significant GGIs in the three different methods are shown
Table 2 False positive rate of the GBIGM in different sample sizes

\begin{tabular}{llllll}
\hline & \multicolumn{5}{c}{ Sample size } \\
\cline { 2 - 6 } Model & 1000 & 2000 & 3000 & 4000 & 5000 \\
\hline Dominant-dominant model & 0.02 & 0.05 & 0.05 & 0.05 & 0.03 \\
Recessive-dominant model & 0.04 & 0.04 & 0.06 & 0.05 & 0.04 \\
Recessive-recessive model & 0.05 & 0.04 & 0.04 & 0.04 & 0.04 \\
A special interaction model & 0.07 & 0.05 & 0.05 & 0.06 & 0.04 \\
Additive-additive model & 0.80 & 0.78 & 0.78 & 0.77 & 0.76 \\
Multiplicative-multiplicative model & 0.04 & 0.05 & 0.04 & 0.06 & 0.05 \\
Exclusive OR model & 0.08 & 0.06 & 0.04 & 0.06 & 0.04 \\
Threshold model & 0.09 & 0.08 & 0.06 & 0.05 & 0.08 \\
\hline
\end{tabular}

Table 3 The description of genes and SNP numbers in RA data

\begin{tabular}{lcc}
\hline Gene & Chromsome & SNP number \\
\hline PADI1 & 1 & 3 \\
PADI2 & 1 & 7 \\
PADI4 & 1 & 5 \\
PADI6 & 1 & 6 \\
PRKD3 & 2 & 7 \\
GC & 4 & 12 \\
GLRX & 5 & 2 \\
CDSN & 6 & 5 \\
PSORS1C1 & 6 & 14 \\
TXNDC5 & 6 & 128 \\
CA1 & 8 & 38 \\
BUB3 & 10 & 3 \\
SORBS1 & 10 & 10 \\
VDR & 12 & 19 \\
SERPINA1 & 14 & 5 \\
PCSK6 & 15 & 74 \\
DNAH9 & 17 & 43 \\
\hline
\end{tabular}

Table 4 The ranks of the five significant GGls in the results of three methods

\begin{tabular}{lcccc}
\hline & & \multicolumn{3}{c}{ Rank } \\
\cline { 3 - 5 } Gene 1 & Gene 2 & GBIGM & KCCU & SBEM \\
\hline GC & PRKD3 & 1 & 33 & 53 \\
PADI1 & PRKD3 & 2 & 38 & 1 \\
CDSN & SERPINA1 & 3 & 24 & 86 \\
BUB3 & PADI4 & 4 & 10 & 118 \\
PADI6 & SERPINA1 & 5 & 13 & 81 \\
\hline
\end{tabular}

in Table 4. Therefore, the gene-based methods, especially the GBIGM method we proposed, have the potential to be more powerful than the SNP-based methods.

\section{DISCUSSION}

The GBIGM we proposed used the information gain as a statistic to detect the interactions between genes in case-control studies. As a nonparametric method, our model could detect both the linear relationship and the nonlinear relationship between two genes. Compared with SBEM and KCCU in the simulated data, the power of GBIGM proposed are larger than the others in the most cases. The method we proposed is stable to the sample size through the test of 
false positive rates. It is a suitable and powerful tool for detecting GGIs for most disease models except recessive-recessive model (other methods are also not suitable) and additive-additive model (high false positive rates). Compared with the other methods, GBIGM can obtain fewer significant results, and it is important for biologists to perform biological verification. We built an online analysis platform of GBIGM for the scientists using (http://nclab.hit.edu.cn/GBIGM).

\section{CONFLICT OF INTEREST}

The authors declare no conflict of interest.

\section{ACKNOWLEDGEMENTS}

This work was supported in part by the National Natural Science Foundation of China (61271346, 61300116, 61172098, 61402132 and 91335112), Specialized Research Fund for the Doctoral Program of Higher Education of China (20112302110040), Fundamental Research Funds for the Central Universities (HIT.KISTP.201418), Natural Science Foundation of Heilongjiang Province (QC2013C063) and the Fund of Heilongiiang Education Department (12531298 and 12531299).

1 Balding DJ: A tutorial on statistical methods for population association studies. Nat Rev Genet 2006; 7: 781-791.

2 Zheng G, Meyer M, Li W, Yang Y: Comparison of two-phase analyses for case-control genetic association studies. Stat Med 2008; 27: 5054-5075.

3 Visscher PM, Hemani G, Vinkhuyzen AA et al: Statistical power to detect genetic (co)variance of complex traits using SNP data in unrelated samples. PLoS Genet 2014; 10: e1004269.

4 Cardon LR, Bell JI: Association study designs for complex diseases. Nat Rev Genet 2001; 2: 91-99.

5 Maher B: Personal genomes: the case of the missing heritability. Nature 2008; 456 : 18-21.

6 Phillips PC: Epistasis - the essential role of gene interactions in the structure and evolution of genetic systems. Nat Rev Genet 2008; 9: 855-867.

7 Fisher RA: The correlation between relatives on the supposition of Mendelian inheritance. Trans R Soc Edinb 1918; 52: 35.

8 Cockerham CC: An extension of the concept of partitioning hereditary variance for analysis of covariances among relatives when epistasis is present. Genetics 1954; 39: 859-882.

9 Kempthorne O: The correlation between relatives in a random mating population. Proc $R$ Soc Lond B Biol Sci 1954; 143: 102-113.

10 Cordell $\mathrm{HJ}$ : Epistasis: what it means, what it doesn't mean, and statistical methods to detect it in humans. Hum Mol Genet 2002; 11: 2463-2468.

11 Schwender $\mathrm{H}$, Ickstadt K: Identification of SNP interactions using logic regression. Biostatistics 2008; 9: 187-198.

12 Dong $\mathrm{C}$, Chu $\mathrm{X}$, Wang $\mathrm{Y}$ et al: Exploration of gene-gene interaction effects using entropy-based methods. Eur J Human Genet 2008; 16: 229-235.

13 Kang G, Yue W, Zhang J, Cui Y, Zuo Y, Zhang D: An entropy-based approach for testing genetic epistasis underlying complex diseases. J Theor Biol 2008; 250: 362-374.
14 Ritchie MD, Hahn LW, Roodi N et al: Multifactor-dimensionality reduction reveals high-order interactions among estrogen-metabolism genes in sporadic breast cancer. Am J Human Genet 2001; 69: 138-147.

15 Zhang Y, Liu JS: Bayesian inference of epistatic interactions in case-control studies. Nat Genet 2007; 39: 1167-1173.

16 Jiang X, Barmada MM, Visweswaran S: Identifying genetic interactions in genome-wide data using Bayesian networks. Genet Epidemiol 2010; 34: 575-581.

17 Chen X, Liu CT, Zhang M, Zhang H: A forest-based approach to identifying gene and gene gene interactions. Proc Natl Acad Sci USA 2007; 104: 19199-19203.

18 Schwarz DF, Konig IR, Ziegler A: On safari to Random Jungle: a fast implementation of random forests for high-dimensional data. Bioinformatics 2010; 26: 1752-1758.

19 Koo CL, Liew MJ, Mohamad MS, Salleh AH: A review for detecting gene-gene interactions using machine learning methods in genetic epidemiology. Biomed Res Int 2013; 2013: 432375

20 Upstill-Goddard R, Eccles D, Fliege J, Collins A: Machine learning approaches for the discovery of gene-gene interactions in disease data. Brief Bioinform 2013; 14: 251-260.

21 Peng Q, Zhao J, Xue F: A gene-based method for detecting gene-gene co-association in a case-control association study. Eur J Human Genet 2010; 18: 582-587.

22 Waaijenborg S, Zwinderman AH: Sparse canonical correlation analysis for identifying, connecting and completing gene-expression networks. BMC Bioinformatics 2009; 10: 315.

23 Yuan Z, Gao Q, He Y et al: Detection for gene-gene co-association via kernel canonical correlation analysis. BMC Genet 2012; 13: 83 .

24 Larson NB, Jenkins GD, Larson MC et al: Kernel canonical correlation analysis for assessing gene-gene interactions and application to ovarian cancer. Eur J Human Genet 2014; 22: 126-131.

25 Larson NB, Schaid DJ: A kernel regression approach to gene-gene interaction detection for case-control studies. Genet Epidemiol 2013; 37: 695-703.

26 Zhang X, Yang X, Yuan Z et al: A PLSPM-based test statistic for detecting gene-gene co-association in genome-wide association study with case-control design. PLoS One 2013; 8: e62129.

27 Li F, Zhao J, Yuan Z, Zhang X, Ji J, Xue F: A powerful latent variable method for detecting and characterizing gene-based gene-gene interaction on multiple quantitative traits. BMC Genet 2013; 14: 89.

28 Shannon CE: A mathematical theory of communication. Bell Syst Tech J 1948; 27: 45.

29 Shannon CE, Weaver W: The Mathematical Theory of Communication. Univ of Illinois Press: Champaign, IL, USA: 1949.

30 Thorisson GA, Smith AV, Krishnan L, Stein LD: The International HapMap Project Web site. Genome Res 2005; 15: 1592-1593.

31 International HapMap C, Frazer KA, Ballinger DG et al: A second generation human haplotype map of over 3.1 million SNPs. Nature 2007; 449: 851-861.

$32 \mathrm{Li} \mathrm{W}$, Reich J: A complete enumeration and classification of two-locus disease models. Hum Hered 2000; 50: 334-349.

$33 \mathrm{Li}$ J, Chen Y: Generating samples for association studies based on HapMap data. BMC Bioinformatics 2008; 9: 44.

34 Barrett T, Wilhite SE, Ledoux P et al: NCBI GEO: archive for functional genomics data sets-update. Nucleic Acids Res 2013; 41: D991-D995.

35 Tan Q, Soerensen M, Kruse TA, Christensen K, Christiansen L: A novel permutation test for case-only analysis identifies epistatic effects on human longevity in the FOXO gene family. Aging Cell 2013; 12: 690-694.

36 Berry KJ, Johnston JE, Mielke PW Jr. Analysis of trend: a permutation alternative to the F test. Percept Mot Skills 2011; 112: 247-257.

37 Dunn OJ: Multiple comparisons among means. J Am Statist Assoc 1961; 56: 52-64.

38 Schorderet DF: Using OMIM (On-line Mendelian Inheritance in Man) as an expert system in medical genetics. Am J Med Genet 1991; 39: 278-284.

39 McKusick VA: Mendelian inheritance in man and its online version, OMIM. Am J Hum Genet 2007; 80: 588-604.

Supplementary Information accompanies this paper on European Journal of Human Genetics website (http://www.nature.com/ejhg) 